\title{
Higher order Borel-Pompeiu representations in Clifford analysis
}

\author{
Juan Bory Reyes ${ }^{a}$, Hennie De Schepper ${ }^{b}$, Alí Guzmán Adán ${ }^{\mathrm{b} * \dagger}$ and \\ Frank Sommen ${ }^{\mathrm{b}}$
}

\section{Communicated by J. Vigo-Aguiar}

In this paper, we show that a higher order Borel-Pompeiu (Cauchy-Pompeiu) formula, associated with an arbitrary orthogonal basis (called structural set) of a Euclidean space, can be extended to the framework of generalized Clifford analysis. Furthermore, in lower dimensional cases, as well as for combinations of standard structural sets, explicit expressions of the kernel functions are derived. Copyright $\odot 2015$ John Wiley \& Sons, Ltd.

Keywords: Borel-Pompeiu representation; Cauchy-Riemann operator; Clifford analysis; higher order Cauchy-type integral; higher order Téodorescu transform; structural set

\section{Introduction and preliminaries}

Clifford analysis in its basic form [1] deals with the study of so-called monogenic functions, that is, null solutions of the Cauchy-Riemann operator, which are an appropriate higher-dimensional analog of holomorphic functions in the complex plane. One of the key results in this function theory is the Borel-Pompeiu representation formula, as is the case in the theory of holomorphic functions in the complex plane, leading in the present context to various higher-dimensional analogs of classical theorems.

Integral representation formulae of Borel-Pompeiu type, expressing Clifford algebra valued functions, play an essential role in solving certain kinds of boundary value problems for partial differential equations related to the Cauchy-Riemann operator. During the last decades, there has been much interest to study such higher-order representation formulae, see for example [2-10]. However, all these results only consider the standard orthogonal basis, whereas in this paper, we generalize this setting to an arbitrary orthogonal basis (or structural set) of Euclidean space and establish a higher-order Borel-Pompeiu (or Cauchy-Pompeiu) formula in that setting. Furthermore, in low dimensional cases, explicit expressions of the kernel functions are derived, also for combinations of standard structural sets.

Let $\left\{e_{1}, e_{2}, \ldots, e_{n}\right\}$ be an orthonormal basis of $\mathbb{R}^{n}$, underlying the construction of the $2^{n}$ dimensional real Clifford algebra $\mathbb{R}_{0, n}$, according to the multiplication rules

$$
e_{i} e_{j}+e_{j} e_{i}=-2 \delta_{i j}
$$

where $\delta_{i j}$ is Kronecker's symbol. Then for $A=\left\{h_{1}, \ldots, h_{k}\right\}\left(1 \leq h_{1}<\cdots<h_{k} \leq n\right)$, the elements $e_{A}=e_{h_{1}} \cdots e_{h_{k}}$ together with $e_{\emptyset}=1$ define a basis of $\mathbb{R}_{0, n}$, whence any $a \in \mathbb{R}_{0, n}$ may be written as $a=\sum_{A} a_{A} e_{A}$, where $a_{A} \in \mathbb{R}$ or still as $a=\sum_{k=0}^{n}[a]_{k}$, where $[a]_{k}=\sum_{|A|=k} a_{A} e_{A}$ is a so-called $k$-vector. If we denote the space of $k$-vectors by $\mathbb{R}_{0, n}^{(k)}$, then

$$
\mathbb{R}_{0, n}=\bigoplus_{k=0}^{n} \mathbb{R}_{0, n}^{(k)} .
$$

In this way, the spaces $\mathbb{R}$ and $\mathbb{R}^{n}$ are identified with $\mathbb{R}_{0, n}^{(0)}$ and $\mathbb{R}_{0, n}^{(1)}$.

Each element $x=\left(x_{0}, x_{1}, \ldots, x_{n}\right) \in \mathbb{R}^{n+1}$ may then be rewritten as

$$
x=x_{0}+\sum_{i=1}^{n} x_{i} e_{i} \in \mathbb{R}_{0, n}^{(0)} \oplus \mathbb{R}_{0, n}^{(1)}
$$

a ESIME-Zacatenco. Instituto Politécnico Nacional. México, DF 07738. México

${ }^{b}$ Clifford Research Group, Department of Mathematical Analysis, Faculty of Engineering and Architecture, Ghent University, Galglaan 2, 9000 Ghent, Belgium

* Correspondence to: Alí Guzmán Adán, Department of Mathematical Analysis, Faculty of Engineering and Architecture, Ghent University, Galglaan 2, 9000 Ghent, Belgium.

†E-mail:aga@cage.ugent.be 
and is called a paravector. For each such $x \in \mathbb{R}_{0, n}^{(0)} \oplus \mathbb{R}_{0, n}^{(1)}$ it holds that

$$
x \bar{x}=\bar{x} x=x_{0}^{2}+x_{1}^{2}+\cdots+x_{n}^{2}=|x|^{2} .
$$

The extension of (1) to a norm on $\mathbb{R}_{0, n}$ is straightforward and leads to

$$
|a|^{2}=[a \bar{a}]_{0}=[\bar{a} a]_{0}=\sum_{A} a_{A}^{2}
$$

Here, the (Clifford) conjugation has been used, which is defined by $\bar{a}=\sum_{A} a_{A} \bar{e}_{A}$, where

$$
\bar{e}_{A}:=(-1)^{k} e_{h_{k}} \cdots e_{h_{1}}=(-1)^{\frac{k(k+1)}{2}} e_{A}
$$

when $e_{A}=e_{h_{1}} \cdots e_{h_{k}}$. The following properties of the norm and the conjugation in a Clifford algebra are well-known, see for instance [11].

Proposition 1

For any $a, b \in \mathbb{R}_{0, n}$, it holds that

(1) $\overline{a b}=\bar{b} \bar{a}$,

(2) $|\bar{a}|=|-a|=|a|$,

(3) $[a \bar{b}]_{0}=[\bar{a} b]_{0}=\langle a, b\rangle$, where $\langle\cdot, \cdot\rangle$ denotes the standard inner product in $\mathbb{R}^{2^{n}}$,

(4) $|a b| \leq 2^{n / 2}|a||b|$,

(5) if $b \neq 0$ is such that $b \bar{b}=|b|^{2}$, then $b$ is invertible and $|a b|=|b a|=|a||b|$.

Let $\Omega$ be a bounded domain in $\mathbb{R}^{n+1}$, with sufficiently smooth boundary $\Gamma$. We will consider functions $f: \Omega \rightarrow \mathbb{R}_{0, n}$, which may be written as

$$
f(x)=\sum_{A} f_{A}(x) e_{A}
$$

with $f_{A} \mathbb{R}$-valued, whence properties such as continuity, differentiability, integrability, and so on, are attributed componentwise. In particular, we define in this way, for any suitable subset $E$ of $\mathbb{R}^{n+1}$, the following sets:

- the set $C^{k}\left(E, \mathbb{R}_{0, n}\right)$ of $\mathbb{R}_{0, n}$-valued, $k$ times continuously differentiable functions on $E$;

- the set $C^{\infty}\left(E, \mathbb{R}_{0, n}\right):=\bigcap_{k=0}^{\infty} C^{k}\left(E, \mathbb{R}_{0, n}\right)$.

For $f \in C^{k}\left(E, \mathbb{R}_{0, n}\right)$, we will write

$$
D_{\alpha} f=\frac{\partial^{|\alpha|} f}{\partial x_{0}^{\alpha_{0}} \cdots \partial x_{n}^{\alpha_{n}}}, \quad|\alpha| \leq k,
$$

where $\alpha=\left(\alpha_{0}, \ldots, \alpha_{n}\right) \in(\mathbb{N} \cup\{0\})^{n+1}$ is a multi-index and $|\alpha|=\alpha_{0}+\cdots+\alpha_{n}$. Furthermore, we consider

- the set $C^{0, \mu}\left(E, \mathbb{R}_{0, n}\right)(\mu \in(0,1])$ of $\mathbb{R}_{0, n}$-valued, $\mu$-Hölder continuous functions on $E$;

- the set $C^{k, \mu}\left(E, \mathbb{R}_{0, n}\right)(k \in \mathbb{N})$ of functions in $C^{0, \mu}\left(E, \mathbb{R}_{0, n}\right)$ whose partial derivatives $D_{\alpha} f$ belong to $C^{0, \mu}\left(E, \mathbb{R}_{0, n}\right)$ for $|\alpha| \leq k$.

Next, let $\psi:=\left\{\psi^{0}, \psi^{1}, \ldots, \psi^{n}\right\} \subset \mathbb{R}_{0, n}^{(0)} \oplus \mathbb{R}_{0, n}^{(1)}$ and denote $\bar{\psi}:=\left\{\overline{\psi^{0}}, \overline{\psi^{1}}, \ldots, \overline{\psi^{n}}\right\}$. Then, we define the left and the right $\psi$-CauchyRiemann operator by their respective actions on functions $f \in C^{1}\left(\Omega, \mathbb{R}_{0, n}\right)$ :

$$
\psi_{D}[f]:=\sum_{i=0}^{n} \psi^{i} \frac{\partial f}{\partial x_{i}}, \quad D^{\psi}[f]:=\sum_{i=0}^{n} \frac{\partial f}{\partial x_{i}} \psi^{i} .
$$

It is easy to prove that the equalities

$$
{ }^{\psi} D^{\bar{\psi}} D={ }^{\bar{\psi}} D^{\psi} D=D^{\psi} D^{\bar{\psi}}=D^{\bar{\psi}} D^{\psi}=\Delta_{n+1}
$$

hold, where $\Delta_{n+1}$ is the $(n+1)$-dimensional Laplace operator, if and only if

$$
\psi^{i} \overline{\psi^{j}}+\psi^{j} \overline{\psi^{i}}=2 \delta_{i j}, \quad i, j=0, \ldots, n .
$$

Note that last equality yields

$$
2 \delta_{i j}=\psi^{i} \overline{\psi^{j}}+\psi^{j} \overline{\psi^{i}}=\psi^{i} \overline{\psi^{j}}+\overline{\psi^{i} \overline{\psi^{j}}}=2\left[\psi^{i} \overline{\psi^{j}}\right]_{0}=2\left\langle\psi^{i}, \psi^{j}\right\rangle_{\mathbb{R}^{n+1}},
$$

whence (3) holds if and only if $\psi$ represents an orthonormal basis of $\mathbb{R}^{n+1} \cong \mathbb{R}_{0, n}^{(0)} \oplus \mathbb{R}_{0, n}^{(1)}$. 
Any set $\psi$ showing the property (4) is called a structural set; it is clear that $\psi$ and $\bar{\psi}$ are structural sets simultaneously. The standard basis of $\mathbb{R}^{n+1}$ given by $\psi_{s t}:=\left\{1, e_{1}, \ldots, e_{n}\right\}$ thus can be considered as the particular structural set generating the standard CauchyRiemann operator $D[f]:=\psi_{\text {st }} D[f]$.

Basic properties of structural sets can be found in $[12,13]$ and the references therein. In particular, the following properties connect the left and right Cauchy-Riemann operators and can be obtained by direct calculation.

Lemma 1

Let $\psi$ and $\varphi$ be two structural sets. Then it holds that

(1) ${ }^{\psi} D[f]=\overline{D^{\bar{\psi}}[\bar{f}]}$, and $D^{\psi}[f]=\overline{\bar{\psi} D[\bar{f}]}$.

(2) $D^{\varphi} \psi D={ }^{\psi} D D^{\varphi}$ in $C^{2}\left(\Omega, \mathbb{R}_{0, n}\right)$.

For fixed $\psi$ and $\Omega$, we introduce the spaces of functions

$$
\psi_{\mathfrak{M}}\left(\Omega, \mathbb{R}_{0, n}\right):=\operatorname{ker}^{\psi} D=\left\{f \in C^{1}\left(\Omega, \mathbb{R}_{0, n}\right):{ }^{\psi} D[f]=0 \text { in } \Omega\right\},
$$

and

$$
\mathfrak{M}^{\psi}\left(\Omega, \mathbb{R}_{0, n}\right):=\operatorname{ker} D^{\psi}=\left\{f \in C^{1}\left(\Omega, \mathbb{R}_{0, n}\right): D^{\psi}[f]=0_{\Omega}\right\},
$$

the elements of which are called $\psi$-hyperholomorphic functions (left and right, respectively).

It is of central importance in the development of a function theory for $\psi$-hyperholomorphic functions to establish the fundamental solution of the operators ${ }^{\psi} D$ and $D^{\psi}$, also called the Cauchy kernel. To this end, we first consider the fundamental solution $\Theta_{n+1}^{(k)}$ of the iterated Laplace operator $\Delta_{n+1}^{k}, k \in \mathbb{N}$, given by

$$
\Theta_{n+1}^{(k)}(x)=\frac{|x|^{2 k-(n+1)}}{\left|\mathbb{S}^{n}\right| 2^{k-1}(k-1) ! \prod_{v=1}^{k}(2 v-n-1)} .
$$

In view of (3), the Cauchy kernel may then be obtained as:

$$
K_{\psi}(x):={ }^{\bar{\psi}} D\left[\Theta_{n+1}^{(1)}\right](x)=D^{\bar{\psi}}\left[\Theta_{n+1}^{(1)}\right](x)=\frac{x_{\bar{\psi}}}{\left|\mathbb{S}^{n}\right||x|^{n+1}},
$$

where

$$
x_{\bar{\psi}}:=\sum_{i=0}^{n} x_{i} \overline{\psi^{i}} \quad \text { if } x=\sum_{i=0}^{n} x_{i} e_{i}
$$

and $\left|\mathbb{S}^{n}\right|$ is the area of the unit sphere $\mathbb{S}^{n}$ in $\mathbb{R}^{n+1}$.

The kernel $K_{\psi}$ has the following important properties:

(1) $K_{\psi} \in C^{\infty}\left(\mathbb{R}^{n+1} \backslash\{0\}, \mathbb{R}_{0, n}\right)$.

(2) $K_{\psi} \in \psi \mathfrak{M}\left(\mathbb{R}^{n+1} \backslash\{0\}, \mathbb{R}_{0, n}\right) \cap \mathfrak{M}^{\psi}\left(\mathbb{R}^{n+1} \backslash\{0\}, \mathbb{R}_{0, n}\right)$.

A first important result then is given by Stokes' formula, which takes, for an arbitrary structural set $\psi$, the following form. For $f, g \in$ $C^{1}\left(\Omega, \mathbb{R}_{0, n}\right) \cap C^{0}\left(\bar{\Omega}, \mathbb{R}_{0, n}\right)$ it holds that

$$
\int_{\Gamma} g(\xi) n_{\psi}(\xi) f(\xi) d S_{\xi}=\int_{\Omega}\left(D^{\psi}[g](\xi) f(\xi)+g(\xi){ }^{\psi} D[f](\xi)\right) d \xi
$$

where

$$
n_{\psi}(\xi)=\sum_{i=0}^{n} n_{i}(\xi) \psi^{i}
$$

with $n_{i}(\xi)$ the $i$-th component of the outward unit normal vector on $\Gamma$ at the point $\xi \in \Gamma$. This Stokes' formula immediately leads to two important consequences, which are widely known and can be found, for the standard setting, in many sources.

Theorem 1

(Cauchy integral theorem). Let $f \in \psi \mathfrak{M}\left(\Omega, \mathbb{R}_{0, n}\right) \cap C^{0}\left(\bar{\Omega}, \mathbb{R}_{0, n}\right)$ and $g \in \mathfrak{M}^{\psi}\left(\Omega, \mathbb{R}_{0, n}\right) \cap C^{0}\left(\bar{\Omega}, \mathbb{R}_{0, n}\right)$. Then it holds that

$$
\int_{\Gamma} g(\xi) n_{\psi}(\xi) f(\xi) d S_{\xi}=0
$$

Theorem 2

(Borel-Pompeiu (Cauchy-Green) formula). Let $f \in C^{1}\left(\Omega, \mathbb{R}_{0, n}\right) \cap C^{0}\left(\bar{\Omega}, \mathbb{R}_{0, n}\right)$. Then it holds that

$$
\int_{\Gamma} K_{\psi}(\xi-x) n_{\psi}(\xi) f(\xi) d S_{\xi}-\int_{\Omega} K_{\psi}(\xi-x)^{\psi} D[f](\xi) d \xi= \begin{cases}f(x) & \text { if } x \in \Omega \\ 0 & \text { if } x \in \mathbb{R}^{n+1} \backslash \bar{\Omega}\end{cases}
$$


The Cauchy kernel thus generates the following two important integrals:

$$
{ }^{\psi} T_{\Omega}[f](x):=-\int_{\Omega} K_{\psi}(\xi-x) f(\xi) d \xi, \quad x \in \mathbb{R}^{n+1},
$$

and

$$
{ }^{\psi} K_{\Gamma}[f](x):=\int_{\Gamma} K_{\psi}(\xi-x) n_{\psi}(\xi) f(\xi) d S_{\xi}, \quad x \notin \Gamma
$$

The first is a generalization of the usual Téodorescu transform; the second represents the usual Cauchy type integral. Note that the $\psi$-Téodorescu transform is the right-inverse to the operator ${ }^{\psi} D$ [14].

Theorem 3

For every $f \in L_{p}\left(\Omega, \mathbb{R}_{0, n}\right), p \in(1, \infty)$, we have

$$
{ }^{\psi} D^{\psi} T_{\Omega}[f](x)= \begin{cases}f(x) & \text { if } x \in \Omega \\ 0 & \text { if } x \in \mathbb{R}^{n+1} \backslash \bar{\Omega}\end{cases}
$$

\section{Iterated Cauchy-Riemann operators}

The main goal of this section is to study the properties of the fundamental solution of so-called iterated Cauchy-Riemann operators, which are obtained by the composition of $k$ generalized Cauchy-Riemann operators associated to different structural sets.

Let $\Psi:=\left\{\psi_{1}, \psi_{2}, \ldots, \psi_{k}\right\}$ be a collection of $k$ structural sets of $\mathbb{R}_{0, n}$ and denote $\bar{\Psi}:=\left\{\bar{\psi}_{k}, \bar{\psi}_{k-1}, \ldots, \bar{\psi}_{1}\right\}$. Then the left and right $\Psi$-iterated Cauchy-Riemann operators are defined as:

$$
{ }^{\Psi} D={ }^{\psi_{k}} D^{\psi_{k-1}} D \ldots{ }^{\psi_{1}} D, \quad D^{\Psi}=D^{\psi_{k}} D^{\psi_{k-1}} \cdots D^{\psi_{1}}
$$

Note that as a consequence of (3), we have

$$
{ }^{\Psi} D^{\bar{\Psi}} D={ }^{\Psi} D^{\Psi} D=D^{\Psi} D^{\bar{\Psi}}=D^{\bar{\Psi}} D^{\Psi}=\Delta_{n+1}^{k}
$$

It then is easy to see that

$$
K_{\Psi}(x):=\bar{\Psi} D\left[\Theta_{n+1}^{(k)}\right]=\bar{\psi}_{1} D^{\bar{\psi}_{2}} D \ldots \bar{\psi}_{k} D\left[\Theta_{n+1}^{(k)}\right]
$$

constitutes a fundamental solution to these iterated Cauchy-Riemann operators.

Given a collection of structural sets $\Psi$, we will define the subcollections

$$
\Psi^{i}=\left\{\psi_{1}, \psi_{2}, \ldots, \psi_{i}\right\} \subset \Psi,
$$

where $1 \leq i \leq k$. Note that $\Psi^{k}=\Psi$. Then the following properties hold.

Proposition 2

(i) $K_{\Psi}(x) \in C^{\infty}\left(\mathbb{R}^{n+1} \backslash\{0\}, \mathbb{R}_{0, n}\right)$.

(ii) $K_{\Psi}(x)=\frac{P_{\Psi}(x)}{\left|\mathbb{S}^{n}\right| 2^{k-1}(k-1) !|x|^{n+1}}$, where $P_{\Psi}(x)$ is an homogeneous polynomial of degree $k$.

(iii) ${ }^{\psi_{1}} D\left[K_{\Psi}\right]=K_{\tilde{\Psi}}$ where $\tilde{\Psi}=\left\{\psi_{2}, \ldots, \psi_{k}\right\}$.

(iv) $K_{\Psi}=D^{\bar{\psi}_{k}} \ldots D^{\bar{\psi}_{2}} D^{\bar{\psi}_{1}}\left[\Theta_{n+1}^{(k)}\right]$.

(v) $D^{\psi_{k}}\left[K_{\Psi}\right]=K_{\Psi^{k-1}}$.

Proof

(ii) As a first step, we show that, for $i=0,1, \ldots k-1$ and $m \in \mathbb{N}$ fixed:

$$
\bar{\psi}_{k-i} D \ldots{\overline{\psi_{k-1}}} D^{\bar{\psi}_{k}} D\left[|x|^{m}\right]=\prod_{v=0}^{i}(m-2 v)|x|^{m-2(i+1)} Q_{\Psi}^{(i)}(x),
$$

where $Q_{\Psi}^{(i)}(x)$ is a homogeneous polynomial of degree $i+1$. Indeed, for $i=0$, we have that $\bar{\psi}_{k} D\left[|x|^{m}\right]=m x \bar{\psi}_{k}|x|^{m-2}$, whence (9) is fulfilled in this case. Next, we proceed by induction: suppose that (9) holds for $i=1, \ldots, j(j<k-1)$ then, for $i=j+1$, we have: 


$$
\begin{aligned}
\bar{\psi}_{k-(j+1)} D \cdot \bar{\psi}_{k-j} D \ldots \bar{\psi}_{k} D\left[|x|^{m}\right] & =\prod_{v=0}^{j}(m-2 v) \bar{\psi}_{k-(j+1)} D\left[|x|^{m-2(j+1)} Q_{\Psi}^{(j)}(x)\right] \\
& =\prod_{v=0}^{j}(m-2 v)\left(\bar{\psi}_{k-(j+1)} D\left[|x|^{m-2(j+1)}\right] Q_{\Psi}^{(j)}(x)+\bar{\psi}_{k-(j+1)} D\left[Q_{\Psi}^{(j)}\right](x)|x|^{m-2(j+1)}\right) \\
& =\prod_{v=0}^{j+1}(m-2 v)\left(x_{\bar{\psi}_{k-(j+1)}}|x|^{m-2(j+2)} Q_{\Psi}^{(j)}(x)+\frac{\bar{\psi}_{k-(j+1)} D\left[Q_{\Psi}^{(j)}\right](x)|x|^{m-2(j+1)}}{m-2(j+1)}\right) \\
& =\prod_{v=0}^{j+1}(m-2 v)|x|^{m-2(j+2)} Q_{\Psi}^{(j+1)}(x),
\end{aligned}
$$

where

$$
Q_{\Psi}^{(j+1)}(x)=x_{\bar{\psi}_{k-(j+1)}} Q_{\Psi}^{(j)}(x)+|x|^{2} \frac{\bar{\psi}_{k-(j+1)} D\left[Q_{\Psi}^{(j)}\right](x)}{m-2(j+1)}
$$

clearly is a homogeneous polynomial of degree $j+2$.

Now putting $i=k-1$ in $(9), m=2 k-(n+1)$ and $P_{\Psi}(x):=Q_{\Psi}^{(k-1)}(x)$, we obtain:

$$
\begin{aligned}
K_{\Psi}(x) & =\frac{\bar{\psi}_{1} D^{\bar{\psi}_{2}} D \ldots \bar{\psi}_{k} D\left[|x|^{2 k-(n+1)}\right]}{\left|\mathbb{S}^{n}\right| 2^{k-1}(k-1) ! \prod_{v=1}^{k}(2 v-n-1)} \\
& =\frac{\prod_{v=0}^{k-1}(2(k-v)-n-1)|x|^{-(n+1)} P_{\Psi}(x)}{\left|\mathbb{S}^{n}\right| 2^{k-1}(k-1) ! \prod_{v=1}^{k}(2 v-n-1)}=\frac{P_{\Psi}(x)}{\left|\mathbb{S}^{n}\right| 2^{k-1}(k-1) !|x|^{n+1}} .
\end{aligned}
$$

(iii) Taking into account (8) and (i), we obtain

$$
\begin{aligned}
{ }^{\psi_{1}} D\left[K_{\Psi}\right] & =\Delta_{n+1} \bar{\psi}_{2} D \ldots \bar{\psi}^{{ }_{k}} D\left[\Theta_{n+1}^{(k)}\right] \\
& =\bar{\psi}_{2} D \ldots \bar{\psi}^{{ }_{k}} D \Delta_{n+1}\left[\Theta_{n+1}^{(k)}\right]=\bar{\psi}_{2} D \ldots \bar{\psi} D\left[\Theta_{n+1}^{(k-1)}\right]=K_{\tilde{\Psi}} .
\end{aligned}
$$

(iv) Because $\Theta_{n+1}^{(k)}$ is a real valued function, we can write $\bar{\psi}_{k} D\left[\Theta_{n+1}^{(k)}\right]=D^{\bar{\psi}_{k}}\left[\Theta_{n+1}^{(k)}\right]$. Then successively applying Lemma 1 (ii), we obtain

$$
\begin{aligned}
K_{\Psi}(x) & =\bar{\psi}_{1} D^{\bar{\psi}_{2}} D \ldots \bar{\psi}_{k} D\left[\Theta_{n+1}^{(k)}\right]={ }^{\bar{\psi}_{1}} D^{\bar{\psi}_{2}} D \ldots \bar{\psi}_{k-1} D D^{\bar{\psi}_{k}}\left[\Theta_{n+1}^{(k)}\right] \\
& =D^{\bar{\psi}_{k} \bar{\psi}_{1}} D^{\bar{\psi}_{2}} D \ldots \bar{\psi}_{k-1} D\left[\Theta_{n+1}^{(k)}\right]=D^{\bar{\psi}_{k}} D^{\bar{\psi}_{k-1}} \bar{\psi}_{1} D \ldots \bar{\psi}_{k-2} D\left[\Theta_{n+1}^{(k)}\right] \\
& =\ldots \\
& =D^{\bar{\psi}_{k}} D^{\bar{\psi}_{k-1}} \ldots D^{\bar{\psi}_{1}}\left[\Theta_{n+1}^{(k)}\right] .
\end{aligned}
$$

(v) From (iv), it follows that

$$
\begin{aligned}
D^{\psi_{k}}\left[K_{\Psi}\right] & =D^{\psi_{k}} D^{\bar{\psi}_{k}} D^{\bar{\psi}_{k-1}} \ldots D^{\bar{\psi}_{1}}\left[\Theta_{n+1}^{(k)}\right]=D^{\bar{\psi}_{k-1}} \ldots D^{\bar{\psi}_{1}} \Delta_{n+1}\left[\Theta_{n+1}^{(k)}\right] \\
& =D^{\bar{\psi}_{k-1}} \ldots D^{\bar{\psi}_{1}}\left[\Theta_{n+1}^{(k-1)}\right]=K_{\Psi^{k-1}} .
\end{aligned}
$$

\section{Higher order Borel-Pompeiu representations}

Given a collection of structural sets $\Psi$, we will now define integral operators generalizing the classical concepts of a Cauchy type integral and a Téodorescu transform.

- $\Psi$-Cauchy type integral:

$$
{ }^{\Psi} K_{\Gamma}[f](x):=\int_{\Gamma} K_{\Psi}(\xi-x) n_{\psi_{k}}(\xi) f(\xi) d S_{\xi} \quad x \notin \Gamma ;
$$


- $\Psi$-Téodorescu transform:

$$
{ }^{\Psi} T_{\Omega}[f](x):=(-1)^{k} \int_{\Omega} K_{\Psi}(\xi-x) f(\xi) d \xi, \quad x \in \mathbb{R}^{n+1}
$$

Observe that, for $x \in \bar{\Omega}$ the integral ${ }^{\Psi} T_{\Omega}[f](x)$ is to be understood in the sense of the Cauchy principal value. Yet, the existence of this integral for every $x \in \mathbb{R}^{n+1}$ still has to be carefully proven.

Theorem 4

Let $f \in L_{1}\left(\Omega, \mathbb{R}_{0, n}\right)$. Then the integral ${ }^{\Psi} T_{\Omega}[f](x)$ exists for every $x \in \mathbb{R}^{n+1}$.

Proof

If $x \in \mathbb{R}^{n+1} \backslash \bar{\Omega}$, then $K_{\Psi}(\xi-x)$ is a bounded continuous function of $\xi \in \Omega$, from which the existence of ${ }^{\Psi} T_{\Omega}[f]$ in $\mathbb{R}^{n+1} \backslash \bar{\Omega}$ follows. Next, suppose that $x \in \bar{\Omega}$. Then for every $\xi \in \Omega(\xi \neq x)$, we obtain, in view of Propositions 1 and 2, the following estimation:

$$
\left|K_{\Psi}(\xi-x) f(\xi)\right| \leq 2^{n / 2}\left|K_{\Psi}(\xi-x)\right||f(\xi)| \leq \frac{2^{n / 2} C_{\Psi}}{\left|\mathbb{S}^{n}\right| 2^{k-1}(k-1) !} \frac{|f(\xi)|}{|\xi-x|^{n+1-k}}
$$

In fact, because

$$
K_{\Psi}(y)=\frac{P_{\Psi}(y)}{\left|\mathbb{S}^{n}\right| 2^{k-1}(k-1) !|y|^{n+1}}
$$

with $P_{\Psi}(x)$ a homogeneous polynomial of degree $k$, there exist a constant $C_{\Psi}>0$ such that

$$
\left|K_{\Psi}(y)\right| \leq \frac{C_{\Psi}|y|^{k}}{\left|\mathbb{S}^{n}\right| 2^{k-1}(k-1) !|y|^{n+1}}
$$

for every $y \in \mathbb{R}^{n+1}(y \neq 0)$. The obtained estimate (10) reduces the problem to the existence of the real integrals

$$
\int_{\Omega} \frac{|f(\xi)|}{|\xi-x|^{n+1-\gamma}} d \xi
$$

for $\gamma>0$ and $f \in L_{1}\left(\Omega, \mathbb{R}_{0, n}\right)$. These integrals have been considered in many sources (see e.g. [15]) and indeed exist for all $\gamma>0$.

Theorem 5

Let $f \in C\left(\bar{\Omega}, \mathbb{R}_{0, n}\right)$. Then it holds that

(i) $\psi_{1} D^{\Psi} T_{\Omega}[f]={ }^{\tilde{\Psi}} T_{\Omega}[f]$ where $\tilde{\Psi}=\left\{\psi_{2}, \ldots, \psi_{k}\right\}, k \geq 2$.

(ii) ${ }^{\Psi} D^{\Psi} T_{\Omega}[f](x)=f(x)$ for every $x \in \Omega$.

Proof

(i) For $k \geq 2$, the singularity of the kernels $K_{\Psi}(\xi-x)$ is not worse than $O\left(\frac{1}{|\xi-x|^{n}}\right)$, allowing for differentiation under the integral of ${ }^{\Psi} T_{\Omega}[f]$. Observe moreover, on account of Proposition 2, that

$$
{ }^{\psi_{1}} D_{x} K_{\Psi}(\xi-x)=(-1) K_{\tilde{\Psi}}(\xi-x)
$$

and in consequence,

$$
{ }^{\psi_{1}} D_{x}{ }^{\Psi} T_{\Omega}[f](x)=(-1)^{k} \int_{\Omega}{ }^{\psi_{1}} D_{x}\left[K_{\Psi}(\xi-x)\right] f(\xi) d \xi=(-1)^{k-1} \int_{\Omega} K_{\tilde{\Psi}}(\xi-x) f(\xi) d \xi={ }^{\tilde{\Psi}} T_{\Omega}[f](x) .
$$

(ii) Applying several times (i), and taking into account Theorem 3, we obtain (ii).

Theorem 6

[Borel-Pompeiu formula of higher order] Let $\Omega \subset \mathbb{R}^{n+1}$ be a bounded domain with sufficiently smooth boundary $\Gamma=\partial \Omega$. Let $\Psi=\left\{\psi_{1}, \psi_{2}, \ldots, \psi_{k}\right\}$ be a collection of $k$ structural sets, $k \in \mathbb{N}$, and let $f \in C^{k}\left(\bar{\Omega}, \mathbb{R}_{0, n}\right)$. Then for $x \in \Omega$ it holds that

$$
f(x)=\sum_{i=1}^{k}(-1)^{i-1} \Psi^{i} K_{\Gamma}^{\Psi^{i-1}} D[f](x)+{ }^{\Psi} T_{\Omega}{ }^{\Psi} D[f](x),
$$

where $\Psi^{0} D[f]=f$. 
Proof

We will proceed by induction on $k$. First, for $k=1$, formula (11) coincides with the classical Borel-Pompeiu formula (7) for the structural set $\psi_{1}$. Next, assume that every function $g \in C^{k-1}\left(\bar{\Omega}, \mathbb{R}_{0, n}\right), k \geq 1$, can be represented as

$$
g=\sum_{i=1}^{k-1}(-1)^{i-1} \Phi^{i} K_{\Gamma}^{\Phi^{i-1}} D[g]+{ }^{\Phi} T_{\Omega}{ }^{\Phi} D[g]
$$

where $\Phi$ is an arbitrary collection of $k-1$ structural sets. Then, given $f \in C^{k}\left(\bar{\Omega}, \mathbb{R}_{0, n}\right)$ and $\Psi=\left\{\psi_{1}, \psi_{2}, \ldots, \psi_{k}\right\}$, we can apply the previous representation to the function ${ }^{\psi_{1}} D[f] \in C^{k-1}\left(\bar{\Omega}, \mathbb{R}_{0, n}\right)$ with the collection $\tilde{\Psi}=\left\{\psi_{2}, \ldots, \psi_{k}\right\}$ :

$$
{ }^{\psi_{1}} D[f]=\sum_{i=1}^{k-1}(-1)^{i-1} \tilde{\Psi}^{i} K_{\Gamma}^{\tilde{\Psi}^{i-1}} D^{\psi_{1}} D[f]+\tilde{\Psi}_{T_{\Omega}} \tilde{\Psi}^{\psi_{1}} D[f]
$$

However, for $2 \leq i \leq k$, we have $\tilde{\Psi}^{i-1} D^{\psi_{1}} D[f]=\left({ }^{\psi_{i}} D \ldots \psi_{2} D\right){ }^{\psi_{1}} D=\Psi^{i} D$, whence

$$
{ }^{\psi_{1}} D[f]=\sum_{i=1}^{k-1}(-1)^{i-1} \tilde{\Psi}^{i} K_{\Gamma}{ }^{\Psi^{i}} D[f]+{ }^{\tilde{\Psi}} T_{\Omega}{ }^{\Psi} D[f]
$$

Now, substituting the previous expression in the classical Borel-Pompeiu formula (11) for the structural set $\psi_{1}$, we obtain

$$
\begin{gathered}
f={ }^{\psi_{1}} K_{\Gamma}[f](x)+{ }^{\psi_{1}} T_{\Omega}^{\psi_{1}} D[f]={ }^{\Psi^{1}} K_{\Gamma}^{\Psi^{0}} D[f]+{ }^{\psi_{1}} T_{\Omega}^{\psi_{1}} D[f] \\
={ }^{\Psi^{1}} K_{\Gamma}^{\Psi^{0}} D[f]+\sum_{i=1}^{k-1}(-1)^{i-1} \psi_{1} T_{\Omega}^{\tilde{\Psi}^{i}} K_{\Gamma}{ }^{\Psi^{i}} D[f]+{ }^{\psi_{1}} T_{\Omega}^{\tilde{\Psi}} T_{\Omega}{ }^{\Psi} D[f] .
\end{gathered}
$$

By Fubini's theorem we have, for $1 \leq i \leq k-1$, that

$$
\begin{aligned}
(-1)^{i-1 \psi_{1}} T_{\Omega} \tilde{\Psi}^{i} K_{\Gamma}{ }^{\Psi^{i}} D[f](x) & =(-1)^{i} \int_{\Omega} K_{\psi_{1}}(\xi-x)\left(\int_{\Gamma} K_{\tilde{\Psi}^{i}}(\tau-\xi) n_{\psi_{i+1}}(\tau)^{\Psi^{i}} D[f](\tau) d S_{\tau}\right) d \xi \\
& =(-1)^{i} \int_{\Gamma}\left(\int_{\Omega} K_{\psi_{1}}(\xi-x) K_{\tilde{\Psi}^{i}}(\tau-\xi) d \xi\right) n_{\psi_{i+1}}(\tau)^{\Psi^{i}} D[f](\tau) d S_{\tau} \\
& =(-1)^{i} \int_{\Gamma} \Psi^{i+1} \phi(x, \tau) n_{\psi_{i+1}}(\tau) \Psi^{\Psi^{i}} D[f](\tau) d S_{\tau}
\end{aligned}
$$

where ${ }^{\Psi^{i+1}} \phi(x, \tau):=\int_{\Omega} K_{\psi_{1}}(\xi-x) K_{\tilde{\Psi}^{i}}(\tau-\xi) d \xi$. However, by classical Borel-Pompeiu formula for $K_{\Psi^{i+1}}(x-\tau)$ and $\psi_{1}$, and in view of Proposition 2 ((ii) and (iii)), we have

$$
\begin{aligned}
K_{\Psi^{i+1}}(x-\tau) & =\int_{\Gamma} K_{\psi_{1}}(\xi-x) n_{\psi_{1}}(\xi) K_{\Psi^{i+1}}(\xi-\tau) d S_{\xi}-\int_{\Omega} K_{\psi_{1}}(\xi-x)^{\psi_{1}} D_{\xi}\left[K_{\Psi^{i+1}}(\xi-\tau)\right] d \xi \\
& ={ }^{\Psi^{i+1}} \tilde{\phi}(x, \tau)-\int_{\Omega} K_{\psi_{1}}(\xi-x) K_{\tilde{\Psi}^{i}}(\xi-\tau) d \xi=\Psi^{i+1} \tilde{\phi}(x, \tau)+(-1)^{i+1} \Psi^{i+1} \phi(x, \tau),
\end{aligned}
$$

where ${ }^{\Psi^{i+1}} \tilde{\phi}(x, \tau)=\int_{\Gamma} K_{\psi_{1}}(\xi-x) n_{\psi_{1}}(\xi) K_{\Psi^{i+1}}(\xi-\tau) d S_{\xi}$, whence we obtain

$$
\Psi^{i+1} \phi(x, \tau)=(-1)^{i+1}\left[K_{\Psi^{i+1}}(x-\tau)-\Psi^{i+1} \tilde{\phi}(x, \tau)\right]
$$

Substituting in (14), we obtain

$$
\begin{aligned}
(-1)^{i-1} \psi_{1} & T_{\Omega}^{\tilde{\Psi}^{i}} K_{\Gamma}{ }^{\Psi^{i}} D[f](x) \\
& =-\int_{\Gamma} K_{\Psi_{i+1}}(x-\tau) n_{\psi_{i+1}}(\tau)^{\Psi^{i}} D[f](\tau) d S_{\tau}+\int_{\Gamma} \Psi^{i+1} \tilde{\phi}(x, \tau) n_{\psi_{i+1}}(\tau)^{\Psi^{i}} D[f](\tau) d S_{\tau} \\
& =(-1)^{i} \Psi^{i+1} K_{\Gamma}^{\Psi^{i}} D[f](x)+\int_{\Gamma} \Psi^{i+1} \tilde{\phi}(x, \tau) n_{\psi_{i+1}}(\tau)^{\Psi^{i}} D[f](\tau) d S_{\tau}
\end{aligned}
$$


and from Stokes formula (5) for $\psi_{i+1}$ it then follows that

$$
\int_{\Gamma} \Psi^{i+1} \tilde{\phi}(x, \tau) n_{\psi_{i+1}}(\tau)^{\Psi^{i}} D[f](\tau) d S_{\tau}=\int_{\Omega}\left(D_{\tau}^{\psi_{i+1}}\left[\Psi^{i+1} \tilde{\phi}(x, \tau)\right] \Psi^{i} D[f](\tau)+\Psi^{i+1} \tilde{\phi}(x, \tau) \Psi^{i+1} D[f](\tau)\right) d \tau
$$

Invoking Proposition 2 (v), we have

$$
\begin{aligned}
D_{\tau}^{\psi_{i+1}}\left[\Psi^{i+1} \tilde{\phi}(x, \tau)\right] & =\int_{\Gamma} K_{\psi_{1}}(\xi-x) n_{\psi_{1}}(\xi) D_{\tau}^{\psi_{i+1}}\left[K_{\Psi^{i+1}}(\xi-\tau)\right] d S_{\xi} \\
& =-\int_{\Gamma} K_{\psi_{1}}(\xi-x) n_{\psi_{1}}(\xi) K_{\Psi^{i}}(\xi-\tau) d S_{\xi}=-\Psi^{i} \tilde{\phi}(x, \tau),
\end{aligned}
$$

whence

$$
\int_{\Gamma} \Psi^{i+1} \tilde{\phi}(x, \tau) n_{\psi_{i+1}}(\tau){ }^{\Psi^{i}} D[f](\tau) d S_{\tau}=\int_{\Omega}\left(\Psi^{i+1} \tilde{\phi}(x, \tau){ }^{\Psi^{i+1}} D[f](\tau)-\Psi^{i} \tilde{\phi}(x, \tau){ }^{\Psi^{i}} D[f](\tau)\right) d \tau
$$

Hence, from (16), we obtain

$$
\begin{aligned}
& \sum_{i=1}^{k-1}(-1)^{i-1} \psi_{1} T_{\Omega}^{\tilde{\Psi}^{i}} K_{\Gamma}{ }^{\Psi^{i}} D[f](x) \\
& =\sum_{i=1}^{k-1}(-1)^{i} \Psi^{i+1} K_{\Gamma}^{\Psi^{i}} D[f](x)+\int_{\Omega}\left[\sum_{i=1}^{k-1}\left(\Psi^{i+1} \tilde{\phi}(x, \tau) \Psi^{i+1} D[f](\tau)-\Psi^{i} \tilde{\phi}(x, \tau) \Psi^{i} D[f](\tau)\right)\right] d \tau \\
& =\sum_{i=1}^{k-1}(-1)^{i} \Psi^{i+1} K_{\Gamma}^{\Psi^{i}} D[f](x)+\int_{\Omega}\left(\Psi^{k} \tilde{\phi}(x, \tau){ }^{\Psi^{k}} D[f](\tau)-\Psi^{1} \tilde{\phi}(x, \tau){ }^{\Psi^{1}} D[f](\tau)\right) d \tau .
\end{aligned}
$$

Now applying Cauchy's integral theorem in the multiple-connected domain $\Omega \backslash(B[x, \epsilon] \cup B[\tau, \epsilon])$ for $x, \tau \in \Omega, x \neq \tau$ and $\epsilon>0$ small, we have

$$
\begin{aligned}
& \Psi^{1} \tilde{\phi}(x, \tau) \\
& =\int_{\Gamma} K_{\psi_{1}}(\xi-x) n_{\psi_{1}}(\xi) K_{\psi_{1}}(\xi-\tau) d S_{\xi} \\
& =\lim _{\epsilon \rightarrow 0}\left(\int_{\partial B(x, \epsilon)} K_{\psi_{1}}(\xi-x) n_{\psi_{1}}(\xi) K_{\psi_{1}}(\xi-\tau) d S_{\xi}+\int_{\partial B(\tau, \epsilon)} K_{\psi_{1}}(\xi-x) n_{\psi_{1}}(\xi) K_{\psi_{1}}(\xi-\tau) d S_{\xi}\right) \\
& =K_{\psi_{1}}(x-\tau)+K_{\psi_{1}}(\tau-x)=0 .
\end{aligned}
$$

Then

$$
\sum_{i=1}^{k-1}(-1)^{i-1} \psi_{1} T_{\Omega}^{\tilde{\Psi}^{i}} K_{\Gamma}{ }^{\Psi^{i}} D[f](x)=\sum_{i=1}^{k-1}(-1)^{i} \Psi^{i+1} K_{\Gamma}{ }^{\Psi^{i}} D[f](x)+\int_{\Omega} \Psi^{\phi}(x, \tau){ }^{\Psi} D[f](\tau) d \tau
$$

On the other hand, (15) yields

$$
\begin{aligned}
{ }^{\psi_{1}} T_{\Omega}^{\tilde{\Psi}} T_{\Omega}{ }^{\Psi} D[f](x) & =(-1)^{k} \int_{\Omega} K_{\psi_{1}}(\xi-x)\left(\int_{\Omega} K_{\Psi^{k-1}}(\tau-\xi) \Psi^{k} D[f](\tau) d \tau\right) d \xi \\
& =(-1)^{k} \int_{\Omega}\left(\int_{\Omega} K_{\psi_{1}}(\xi-x) K_{\Psi^{k-1}}(\tau-\xi) d \xi\right) \Psi^{k} D[f](\tau) d \tau \\
& =(-1)^{k} \int_{\Omega} \Psi^{k} \phi(x, \tau){ }^{\Psi^{k}} D[f](\tau) d \tau \\
& =\int_{\Omega} K_{\Psi}(x-\tau)^{\Psi} D[f](\tau) d \tau-\int_{\Omega} \Psi^{\Psi}(x, \tau)^{\Psi} D[f](\tau) d \tau .
\end{aligned}
$$

Finally, substitution of (18) and (17) in (13) yields (11).

\subsection{Particular cases}

This section is dedicated to some important particular consequences of formula (11). 
Case $k=2$.

In this case, we have

$$
\theta_{n+1}^{(2)}(x)=\frac{|x|^{3-n}}{2(1-n)(3-n)\left|\mathbb{S}^{n}\right|} .
$$

Then for $\Psi=\left\{\psi_{1}, \psi_{2}\right\}$, we obtain

$$
K_{\Psi}(x)=\bar{\psi}_{1} D^{\bar{\psi}_{2}} D\left[\theta_{n+1}^{(2)}\right](x)=\frac{\frac{\sum_{i=1}^{n} \overline{\psi_{1}^{i}} \overline{\psi_{2}^{i}}}{1-n}|x|^{2}+x_{\bar{\psi}_{1}} x_{\bar{\psi}_{2}}}{2\left|\mathbb{S}^{n}\right||x|^{n+1}}=\frac{x_{\bar{\psi}_{1}}\left(x_{\psi_{1}} C_{\psi_{1}, \psi_{2}}+x_{\bar{\psi}_{2}}\right)}{2\left|\mathbb{S}^{n}\right||x|^{n+1}},
$$

where

$$
C_{\psi_{1}, \psi_{2}}:=\frac{\sum_{i=1}^{n} \overline{\psi_{1}^{i}} \overline{\psi_{2}^{i}}}{1-n}
$$

In this case, formula (11) will take the form

$$
f(x)=\int_{\Gamma} K_{\psi_{1}}(\xi-x) n_{\psi_{1}}(\xi) f(\xi) d S_{\xi}-\int_{\Gamma} K_{\Psi}(\xi-x) n_{\psi_{2}}(\xi)^{\psi_{1}} D[f](\xi) d S_{\xi}+\int_{\Omega} K_{\Psi}(\xi-x)^{\psi_{2}} D^{\psi_{1}} D[f](\xi) d \xi .
$$

Case $k=3$.

Here,

$$
\theta_{n+1}^{(3)}(x)=\frac{|x|^{5-n}}{8(1-n)(3-n)(5-n)\left|\mathbb{S}^{n}\right|^{\prime}}
$$

and $\Psi=\left\{\psi_{1}, \psi_{2}, \psi_{3}\right\}$. By straightforward computations, we obtain

$$
K_{\Psi}(x)={ }^{\bar{\psi}_{1}} D^{\bar{\psi}_{2}} D^{\bar{\psi}_{3}} D\left[\theta_{n+1}^{(3)}\right](x)=\frac{\left(x_{\overline{\psi_{1}}} C_{\psi_{2}, \psi_{3}}+\frac{\sum_{i=1}^{n} \overline{\psi_{1}^{i}} x_{\bar{\psi}_{2}} \overline{\psi_{1}^{i}}}{1-n}+C_{\psi_{1}, \psi_{2}} x_{\bar{\psi}_{3}}\right)|x|^{2}+x_{\bar{\psi}_{1}} x_{\bar{\psi}_{2}} x_{\bar{\psi}_{3}}}{8\left|\mathbb{S}^{n}\right||x|^{n+1}},
$$

and from the previous case, we have

$$
K_{\Psi^{2}}(x)=\frac{x_{\bar{\psi}_{1}}\left(x_{\psi_{1}} C_{\psi_{1}, \psi_{2}}+x_{\bar{\psi}_{2}}\right)}{2\left|\mathbb{S}^{n}\right||x|^{n+1}} .
$$

Then

$$
\begin{aligned}
f(x)= & \int_{\Gamma} K_{\psi_{1}}(\xi-x) n_{\psi_{1}}(\xi) f(\xi) d S_{\xi}-\int_{\Gamma} K_{\Psi^{2}}(\xi-x) n_{\psi_{2}}(\xi)^{\psi_{1}} D[f](\xi) d S_{\xi} \\
& +\int_{\Gamma} K_{\Psi}(\xi-x) n_{\psi_{3}}(\xi){ }^{\psi_{2}} D^{\psi_{1}} D[f](\xi) d S_{\xi}-\int_{\Omega} K_{\Psi}(\xi-x)^{\psi_{3}} D^{\psi_{2}} D^{\psi_{1}} D[f](\xi) d \xi
\end{aligned}
$$

Case $k=2 m-1$ and $\Psi=\left\{\psi_{s t}, \overline{\psi_{s t}}, \ldots, \psi_{s t}, \overline{\psi_{s t}}, \psi_{s t}\right\}$.

In this case, ${ }^{\Psi} D=D \Delta_{n+1}^{m-1}$, and ${ }^{\bar{\Psi}} D=\bar{D} \Delta_{n+1}^{m-1}$, whence

$$
K_{\Psi}(x)=\bar{D} \Delta_{n+1}^{m-1}\left[\theta_{n+1}^{(2 m-1)}\right](x)=\bar{D}\left[\theta_{n+1}^{(m)}\right](x)=\frac{\bar{x}|x|^{2(m-1)-(n+1)}}{\left|\mathbb{S}^{n}\right| 2^{m-1}(m-1) ! \prod_{v=1}^{m-1}(2 v-n-1)} .
$$

Case $k=2 m$ and $\Psi=\left\{\psi_{s t}, \overline{\psi_{s t}}, \ldots, \psi_{s t}, \overline{\psi_{s t}}\right\}$.

In this case, ${ }^{\Psi} D=\Delta_{n+1}^{m}={ }^{\Psi} D$, and

$$
K_{\Psi}(x)=\Delta_{n+1}^{m}\left[\theta_{n+1}^{(2 m)}\right](x)=\theta_{n+1}^{(m)}(x)
$$

Taking into account the previous case, we obtain from (11) the following representation for $C^{2 m}\left(\bar{\Omega}, \mathbb{R}_{0, n}\right)$ functions in terms of powers of the Laplacian: 


$$
\begin{aligned}
f(x)= & \sum_{i=1}^{m}\left\{\int_{\Gamma} \frac{(\overline{\xi-x})|\xi-x|^{2(i-1)-(n+1)}}{\left|\mathbb{S}^{n}\right| 2^{i-1}(i-1) ! \prod_{v=1}^{i-1}(2 v-n-1)} n(\xi) \Delta_{n+1}^{i-1}[f](\xi) d S_{\xi}\right. \\
& \left.-\int_{\Gamma} \frac{|\xi-x|^{2 i-(n+1)}}{\left|\mathbb{S}^{n}\right| 2^{i-1}(i-1) ! \prod_{v=1}^{i}(2 v-n-1)} \overline{n(\xi)} D \Delta_{n+1}^{i-1}[f](\xi) d S_{\xi}\right\} \\
& +\int_{\Omega} \frac{|\xi-x|^{2 m-(n+1)}}{\left|\mathbb{S}^{n}\right| 2^{m-1}(m-1) ! \prod_{v=1}^{m}(2 v-n-1)} \Delta_{n+1}^{m}[f](\xi) d \xi
\end{aligned}
$$

This is the same formula as obtained in [2], Theorem 3.

Case $\Psi=\left\{\psi_{s t}, \psi_{s t}, \ldots, \psi_{s t}\right\}$.

In this case, we have ${ }^{\Psi} D=D^{k}$, and $K_{\Psi}(x)=\bar{D}^{k}\left[\theta_{n+1}^{(k)}\right](x)$. Direct computation for $k=1, \ldots, 6$ yields

$$
K_{\Psi}(x)=\bar{D}^{k}\left[\theta_{n+1}^{(k)}\right](x)=\frac{\bar{x}(x+\bar{x})^{k-1}}{\left|\mathbb{S}^{n}\right| 2^{k-1}(k-1) !|x|^{n+1}} .
$$

Hence, in this case and for these values of $k,(11)$ is in accordance with [2] Theorem 1. On the other hand, it is easy to check that

$$
\frac{\bar{x}(x+\bar{x})^{k-1}}{\left|\mathbb{S}^{n}\right| 2^{k-1}(k-1) !|x|^{n+1}}
$$

is a fundamental solution for $D^{k}$ for every $k \in \mathbb{N}$. In view of the previous results for $k=1, \ldots, 6$, we thus conjecture that

$$
\bar{D}^{k}\left[\theta_{n+1}^{(k)}\right](x)=\frac{\bar{x}(x+\bar{x})^{k-1}}{\left|\mathbb{S}^{n}\right| 2^{k-1}(k-1) !|x|^{n+1}}, \quad \text { for every } k \in \mathbb{N} .
$$

If this conjecture is true then, we can conclude that formula (11) is a generalization of formula (2.4), [2].

\section{References}

1. Brackx F, Delanghe R, Sommen F. Clifford Analysis. In Research Notes in Mathematics, Vol. 76. Pitman (Advanced Publishing Program): Boston, 1982.

2. Begehr H. Iterated integral operators in Clifford analysis. Zeitschrift fur Analysis und Ihre Anwendungen 1999; 18:361-377.

3. Begehr H. Representation formulas in Clifford analysis. In: acoustics, mechanics and the related topics of mathematical analysis. World Scientific: Singapore, 2002,8-13.

4. Begehr H. Integral representations in complex, hypercomplex and Clifford analysis. Integral Transforms and Special Functions 2002; 13(3):223-241.

5. Begehr H, Du Jinyuan ZZ. On higher order Cauchy-Pompeiu formula in Clifford analysis and its applications. General Mathematics 2003; 11 (3-4):5-26.

6. Begehr H, Zhongxiang Z, Jinyuan D. On Cauchy-Pompeiu formula for functions with values in a universal Clifford algebra. Acta Mathematica Scientia 2003; 23(1):95-103.

7. Begehr H, Zhongxiang Z, Ngoc Ha VT. Generalized integral representations in Clifford analysis. Complex Variables and Elliptic Equations 2006; 51(8-11):745-762.

8. Gürlebeck K, Zhang Z. Generalized integral representations for functions with values in C (V 3, 3). Chinese Annals of Mathematics, Series B 2011; 32(1):123-138.

9. Zhongxiang Z. On a higher order Cauchy-Pompeiu formula for functions with values in a universal Clifford algebra. Bulletin of the Belgian Mathematical Society-Simon Stevin 2007; 14(1):87-97.

10. Zhongxiang Z. A revised higher order Cauchy-Pompeiu formulas in Clifford analysis and its application. Journal of Applied Functional Analysis 2007; 2(3):269-278.

11. Gürlebeck K, Habetha K, Sprößig W. Holomorphic functions in the plane and n-dimensional space. Springer Science \& Business Media, 2008.

12. Shapiro MV. On some boundary-value problems for functions with values in Clifford algebra. Matematički Vesnik 1988; (103):321-326.

13. Shapiro M. On the conjugate harmonic functions of M. Riesz-E. Stein-G. Weiss. Topics in Complex Analysis, Differential Geometry and Mathematical Physics (St. Konstantin, 1996) 1996:8-32.

14. Blaya RA, Reyes JB, Adán A. G, Kähler U. On the П-operator in Clifford analysis. Journal of Mathematical Analysis and Applications 2016; 434(2): 1138-1159.

15. Hedberg LI. On certain convolution inequalities. Proceedings of the American Mathematical Society 1972; 36(2):505-510. 